\title{
Effect of Nanotube Functionalization on the Elastic Properties of Polyethylene Nanotube Composites
}

\author{
G. M. Odegard* \\ Michigan Technological University, Houghton, Michigan 49931 \\ S. J. V. Frankland ${ }^{\dagger}$ \\ National Institute of Aerospace, Hampton, Virginia 23666 \\ and \\ T. S. Gates \\ NASA Langley Research Center, Hampton, Virginia 23681
}

\begin{abstract}
The effects of the chemical functionalization of a single-wall carbon nanotube in nanotube/polyethylene composites on the bulk elastic properties are presented. Constitutive equations are established for composites containing both functionalized and nonfunctionalized nanotubes using an equivalent-continuum modeling technique. The elastic properties of both composite systems are predicted for amorphous and crystalline polyethylene matrices with various nanotube lengths, volume fractions, and orientations. The results indicate that for the specific composite materials considered in this study most of the elastic stiffness constants of the composite with functionalized nanotubes are either less than or equal to those of the composite without functionalized nanotubes.
\end{abstract}

\section{Nomenclature}

$\begin{array}{ll}\boldsymbol{A}_{f} & =\text { strain concentration tensor of effective fiber } \\ C_{i j m n}, \boldsymbol{C} & =\text { elastic stiffness tensor of the composite } \\ C_{k l}^{f}, \boldsymbol{C}_{f} & =\text { elastic stiffness tensor of effective fiber } \\ C_{k l}^{m}, \boldsymbol{C}_{m} & =\text { elastic stiffness tensor of matrix } \\ \boldsymbol{C}_{0} & =\text { elastic stiffness tensor of reference medium } \\ c_{f} & =\text { effective fiber volume fraction } \\ D^{I} & =\text { molecular mechanics parameter } \\ E, E_{i} & =\text { composite Young's modulus } \\ e & =\text { applied strain } \\ G, G_{k l} & =\text { composite shear modulus } \\ \boldsymbol{I} & =\text { identity tensor } \\ K_{i j}^{f} & =\text { plane-strain bulk modulus of effective fiber } \\ K^{r} & =\text { molecular mechanics parameter } \\ K^{\theta} & =\text { molecular mechanics parameter } \\ R & =\text { molecular mechanics parameter } \\ r^{I} & =\text { molecular mechanics parameter } \\ S & =\text { Eshelby tensor } \\ u_{i}(B) & =\text { displacements applied onto boundary } B \\ V & =\text { volume of effective fiber } \\ x_{i} & =\text { coordinate system of effective fiber } \\ \gamma & =\text { applied shear strain } \\ \delta_{i j} & =\text { Kronecker delta } \\ \varepsilon_{i j} & =\text { strain tensor } \\ \eta_{k l}^{f} & =\text { effective fiber parameter tensor } \\ \Theta & =\text { molecular mechanics parameter } \\ \kappa, \mu & =\text { orientation parameters } \\ \Lambda & =\text { total strain energy of effective fiber } \\ \sigma_{i j} & =\text { stress tensor } \\ & \end{array}$

Presented as Paper 2003-1701 at the AIAA/ASME/ASCE/AHS 44th Structures, Structural Dynamics, and Materials Conference, Norfolk, VA, 7-10 April 2003; received 25 March 2004; revision received 24 February 2005; accepted for publication 28 March 2005. This material is declared a work of the U.S. Government and is not subject to copyright protection in the United States. Copies of this paper may be made for personal or internal use, on condition that the copier pay the $\$ 10.00$ per-copy fee to the Copyright Clearance Center, Inc., 222 Rosewood Drive, Danvers, MA 01923; include the code 0001-1452/05 \$10.00 in correspondence with the CCC.

*Assistant Professor, Department of Mechanical EngineeringEngineering Mechanics. Senior Member AIAA.

${ }^{\dagger}$ Senior Staff Scientist, 144 Research Drive.

† Senior Materials Research Engineer, MS 188E. Associate Fellow AIAA.

\section{Introduction}

INGLE-WALLED carbon nanotube (SWCNT)-reinforced polymer composites have the potential to provide order-ofmagnitude increases in strength and stiffness relative to typical carbon-fiber reinforced polymeric composites. ${ }^{1}$ The remarkable property-to-weight ratio of these materials makes them ideal candidates for aerospace-related structural applications. The mechanical behavior of nanotube/polymer composites depends not only on the individual properties of the polymer and the nanotubes, but also on the nanotube/polymer interaction.

Because of the nanoscale nature of the constituents, a multiscale modeling effort must first address behavior that occurs at the local or constituent level. In this study, the polymer/nanotube interaction is assumed to be confined to a cylindrical region surrounding the SWCNT and extending a finite radius away from the exterior of the tube. The strength or integrity of this nanotube/polymer interaction is defined by the ability to transfer mechanical load between the nanotube and polymer and has been a matter of significant discussion in the literature. ${ }^{2-14}$ In general, two basic approaches have been proposed to improve the strength of the interaction. The first approach involves forming a strong, nonbonded interaction between the polymer and the nanotube without modifying the nanotube structure. This approach assumes a chemical strengthening of the nonbonded interactions with the nanotube ${ }^{15}$ and the improvement of the apparent mechanical connection with the nanotube, for example, by wrapping a large polymer molecule around the nanotube. ${ }^{16,17}$ The second approach requires the formation of a chemical covalent bond between the nanotube and polymer directly, also known as functionalization. There is reasonable evidence for the presence of such nanotube/polymer covalent bonds. ${ }^{18,19}$ Some studies have indicated that functionalization can occur through chemical bonds added to the nanotube sidewalls. ${ }^{3-8}$ Addition of small organic groups to nanotube sidewalls has been reported via in situ generated diazonium compounds ${ }^{4}$ and by fluorination of nanotubes ${ }^{5,6}$ followed by alkylation. ${ }^{7}$ Functionalization can also occur through oxidizing the nanotube sample to induce the formation hydroxyl or carboxylic acid groups at surface defects on the nanotube. ${ }^{9-14}$ This type of functionalization can occur with copolymers, ${ }^{9-11}$ proteins, ${ }^{12}$ organosilanes, ${ }^{13}$ and metal catalysts. ${ }^{14}$ It is known that covalent bonding affects the elastic mechanical properties of the nanotube itself because the formation of a chemical bond with carbon atoms in a nanotube interrupts the $\mathrm{sp}^{2}$ hybridization of the nanotube, thereby forming a site that is closer to an $\mathrm{sp}^{3}$ hybridized carbon. ${ }^{20,21}$ 
However, analysis has not provided a description of how functionalization affects the overall mechanical properties of the composite.

A recent simulation study by one of the authors ${ }^{22}$ has predicted that for carbon nanotube/polyethylene composites there is at least a one-order-of-magnitude increase in the strength for composites with covalent bonding between the nanotube and adjacent polymer molecules, relative to systems without the covalent bonds. However, the hybridization change can weaken the chemical bonds in the vicinity of the functionalization, and this effect should be manifested as a proportional change in the material elastic constants. ${ }^{15,16}$ It is proposed therefore that hierarchical analysis and subsequent predictions of macroscopic, elastic behavior can provide additional insights into the relative merits of nanotube functionalization and the relationship of functionalization to nanotube/polymer bulk mechanical properties.

In the present paper, elastic constitutive models are developed for nanotube/polyethylene composites, with and without functionalization, to predict the effect of functionalization on the elastic mechanical stiffness properties. The constitutive models are established using a recently developed, multiscale modeling method for predicting the elastic properties of nanotube/polymer composites. ${ }^{23,24}$ The equivalent-continuum models are then combined with a micromechanical analysis to predict Young's moduli and the shear moduli of composite systems with amorphous and crystalline polyethylene matrices, various nanotube lengths, volume fractions, and orientations. Results from these analyses are quantified, and the predicted elastic mechanical properties of the functionalized and nonfunctionalized nanotube composite systems are compared.

\section{Materials}

Three materials were used in this study to represent the nanotube/polymer composite. The single-walled carbon nanotube was a $(10,10)$ nanotube of radius $6.78 \AA$. The polymer matrix material immediately adjacent to the nanotube was a crystalline polyethylene. The properties of this material were assumed to be orthotropic and are determined using the method outlined in this paper. The bulk, amorphous polymer matrix material was polyethylene and was assumed to be isotropic, with a representative Young's modulus and Poisson's ratio of $0.9 \mathrm{GPa}$ and 0.3 , respectively. Crystalline polymers are typically composed of crystalline lamellae that are dispersed in an amorphous polymer phase. ${ }^{25}$ Impurities are usually segregated to the amorphous phase. As an approximation, it is assumed in this paper that the crystalline polymer maintains its crystalline structure throughout the entire material and that the nanotube is directly incorporated into the polymer crystal.

\section{Equivalent-Continuum Modeling}

To apply modeling and computer simulation to enhance the development of nanostructured materials systems, it is necessary to consider the structure-property relationships. These relationships relate the intrinsic structure of the material to the desired engineering-level property or performance. Therefore, in spite of the importance of understanding the molecular structure and nature of materials, at some level in the multiscale analysis the behavior of collections of molecules and atoms must be homogenized. At this level, the continuum level, the observed macroscopic behaviour is explained by disregarding the discrete atomistic and molecular structure and assuming that the material is continuously distributed throughout its volume. The continuum material is assumed to have an average density and can be subjected to body forces such as gravity and surface forces such as the contact between two bodies. The current approach for connecting atomistic models to continuum models uses relevant input from the atomistic simulations and attempts to carry forward the critical information that represents the continuum with the intrinsic nanoscale features incorporated as well. This section describes this modeling approach.

A hierarchical modeling scheme based on the equivalentcontinuum modeling technique is used to predict the bulk mechanical behavior of nanostructured materials. ${ }^{23,24}$ In summary, the hierarchical model consists of three major steps. First, a suitable representative volume element (RVE) of the nanostructured material is chosen based on the geometry of a molecular model. Second, an equivalent-truss finite element model of the RVE is developed as an intermediate step to link the molecular and equivalent-continuum models. Finally, an equivalent-continuum model of the RVE is developed in which the molecular potential energy and the total strain energy in the molecular and equivalent-continuum models, respectively, under identical loading conditions, are set equal. The effective mechanical properties of the equivalent continuum are then determined from equating these energies. Each step of the implementation of this method for a functionalized and nonfunctionalized nanotube/polyethylene composite and a pure crystalline polyethylene material is described next. Further details of the modeling method can be found in Refs. 23 and 24.

\section{Molecular Model}

The purpose of the molecular model is to establish the structure of the equilibrated molecular system. Molecular-dynamics (MD) simulations were therefore used to determine the equilibrium structures of the nonfunctionalized nanotube composite, functionalized nanotube composite, and the pure crystalline polymer (left-hand sides of Figs. 1, 2, and 3, respectively). The RVE of a typical nanostructured material is on the nanometer length scale; therefore, the material of the RVE is not considered continuous but is a discrete assemblage of many atoms. The molecular model represents the RVE of the equilibrium molecular structure of the nanostructured material. Interaction of the atoms is described in terms of molecular-mechanics force constants, which are known for most atomic structures. ${ }^{26}$
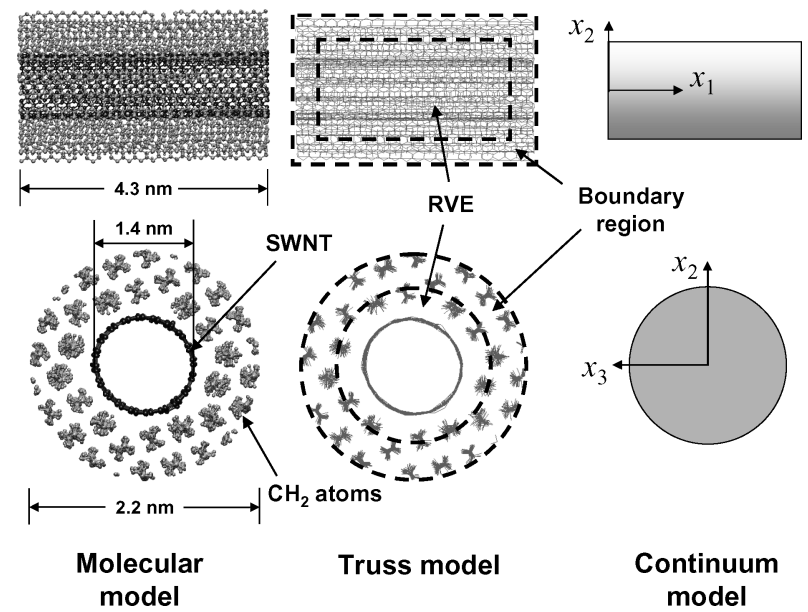

Truss model

$$
\begin{gathered}
\text { Continuum } \\
\text { model }
\end{gathered}
$$

Fig. 1 Equivalent-continuum modeling of nonfunctionalized system. Only the bond-stretch elements are shown in the truss model.
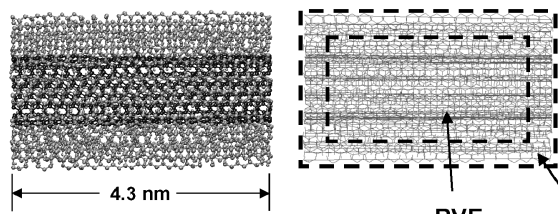

$x_{2}$
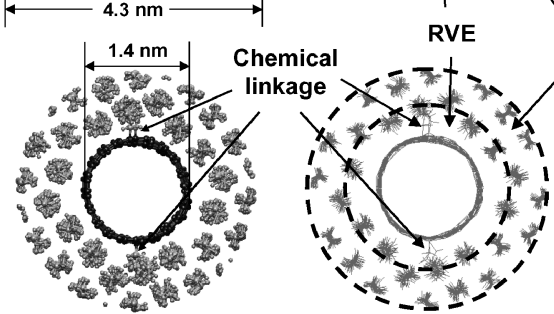

Boundary

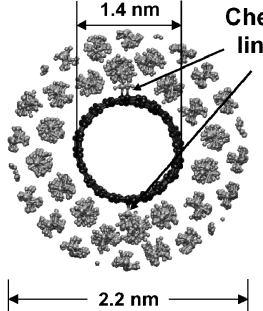

Molecular

Truss model

model region

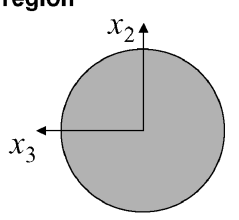

Fig. 2 Equivalent-continuum modeling of functionalized system. Only the bond-stretch elements are shown in the truss model. 


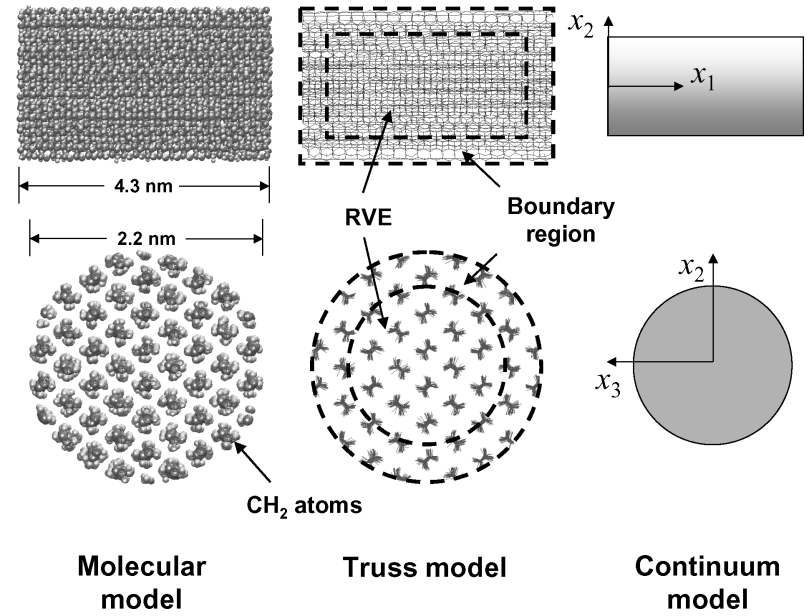

Fig. 3 Equivalent-continuum modeling of crystalline polyethylene. Only the bond-stretch elements are shown in the truss model.

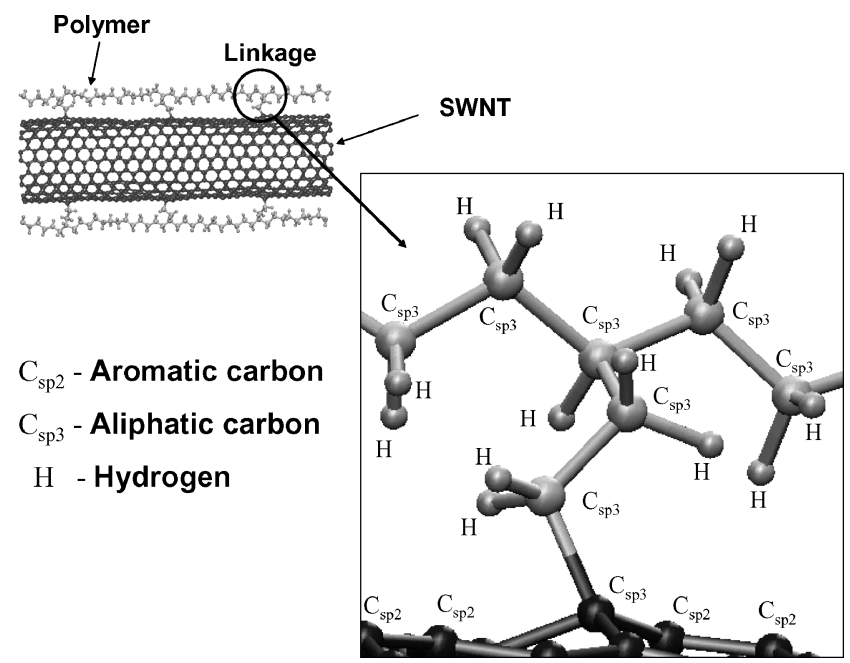

Fig. 4 Modeled atomic structure of the functionalized nanotube/ polyethylene composite.

For both composite systems examined in this study, the starting configuration for the MD simulation was a crystalline polyethylene matrix containing a $(10,10)$ nanotube of radius $6.78 \AA$. To establish the equilibrium molecular structure, the matrix contained 176 chains of $42 \mathrm{CH}_{2}$ units. To establish a simplified, cylindrical RVE geometry, the selected RVEs did not contain all 176 chains, as shown in Figs. 1-3. These chains were aligned and continuous along the entire length of the RVE. For clarity, two views of the molecular models are included in Figs. 1-3 for each material. These views were chosen because of their clear depiction of the molecular structure, as they are aligned with axes of material symmetry. Even though Figs. 1-3 are two dimensional, the actual molecular models were three dimensional. For all three models, the nanotube and polymer chains were replicated across the periodic boundaries of the simulation cell, making them infinitely long in the $x_{1}$ direction. In the composite with nanotube functionalization, two polymer chains were attached to six carbons of the nanotube by chemical linkages consisting of $2 \mathrm{CH}_{2}$ groups. The linkages are presented schematically in Fig. 4. In the MD simulations of the composite, all of the covalent chemical bonds were modeled with the hydrocarbon potential developed by Brenner et al. ${ }^{27}$ The nonbonded interactions between the polymer chains and between the nanotube and the polymer chains were modeled with the Lennard-Jones potential with the parameters used previously. ${ }^{22,28}$ The systems were then equilibrated at $300 \mathrm{~K}$ for $1-2 \mathrm{ps}$ via the MD simulation. The MD simulations were carried out using the DL-POLY ${ }^{\complement}$ (Ref. 29) software.

The equivalent-continuum modeling technique used in this study requires the interaction of the atoms in the molecular model to be
Table 1 Bond-stretching parameters

\begin{tabular}{lcc}
\hline \hline Bond stretching & $R, \AA$ & $K^{r}, \mathrm{kcal} /\left(\right.$ mole $\left.\AA^{2}\right)$ \\
\hline $\mathrm{C}_{\mathrm{sp} 2}-\mathrm{C}_{\mathrm{sp} 2}$ & 1.400 & 469 \\
$\mathrm{C}_{\mathrm{sp} 2}-\mathrm{C}_{\mathrm{sp} 3}$ & 1.510 & 317 \\
$\mathrm{C}_{\mathrm{sp} 3}-\mathrm{C}_{\mathrm{sp} 3}$ & 1.526 & 310 \\
$\mathrm{C}_{\mathrm{sp} 3}-\mathrm{H}$ & 1.090 & 340 \\
\hline \hline
\end{tabular}

Table 2 Bond-angle variation parameters

\begin{tabular}{lcc}
\hline \hline Bond-angle variation & $\Theta$, deg & $K^{\theta}, \mathrm{kcal} /\left(\mathrm{mole} \mathrm{rad}^{2}\right)$ \\
\hline $\mathrm{C}_{\mathrm{sp} 2}-\mathrm{C}_{\mathrm{sp} 2}-\mathrm{C}_{\mathrm{sp} 2}$ & 120.0 & 63 \\
$\mathrm{C}_{\mathrm{sp} 2}-\mathrm{C}_{\mathrm{sp} 2}-\mathrm{C}_{\mathrm{sp} 3}$ & 120.0 & 70 \\
$\mathrm{C}_{\mathrm{sp} 2}-\mathrm{C}_{\mathrm{sp} 3}-\mathrm{C}_{\mathrm{sp} 2}$ & 120.0 & 63 \\
$\mathrm{C}_{\mathrm{sp} 2}-\mathrm{C}_{\mathrm{sp} 3}-\mathrm{C}_{\mathrm{sp} 3}$ & 114.0 & 63 \\
$\mathrm{C}_{\mathrm{sp} 3}-\mathrm{C}_{\mathrm{sp} 3}-\mathrm{C}_{\mathrm{sp} 3}$ & 109.5 & 40 \\
$\mathrm{C}_{\mathrm{sp} 3}-\mathrm{C}_{\mathrm{sp} 3}-\mathrm{H}$ & 109.5 & 50 \\
$\mathrm{H}-\mathrm{C}_{\mathrm{sp} 3}-\mathrm{H}$ & 109.5 & 35 \\
\hline \hline
\end{tabular}

Table 3 Van der Waals interaction parameters

\begin{tabular}{lcc}
\hline \hline Van der Waals interaction & $D^{I}, \mathrm{kcal} / \mathrm{mole}$ & $r^{I}, \AA$ \\
\hline $\mathrm{C}_{\mathrm{sp} 2}$ & 0.0860 & 3.816 \\
$\mathrm{C}_{\mathrm{sp} 3}$ & 0.1094 & 3.816 \\
$\mathrm{H}$ & 0.0157 & 2.974 \\
\hline
\end{tabular}

described in terms of molecular-mechanics force constants. Consequently, the interaction of the atoms in the molecular model was defined in terms of bond stretching, bond-angle variation, and Van der Waals interactions. ${ }^{24}$ The force constants used for both models are shown in Tables 1-3 and were taken from the AMBER force field ${ }^{30}$ in a manner similar to that presented by Odegard et al. ${ }^{24}$ The force constants were assigned with respect to the structure indicated in Fig. 4 in that the atoms labeled as $\mathrm{C}_{\mathrm{sp} 2}, \mathrm{C}_{\mathrm{sp} 3}$, and $\mathrm{H}$ correspond to aromatic carbon atoms $\left(\mathrm{sp}^{2}\right.$ orbital), aliphatic carbon atoms $\left(\mathrm{sp}^{3}\right.$ orbital), and hydrogen atoms that are attached to aliphatic carbon atoms, respectively. In the nonfunctionalized system, all of the carbon atoms in the nanotube were modeled as aromatic carbon atoms.

\section{Truss Model}

The transition from molecular model to continuum is facilitated by the selection of a RVE. The RVE is several nanometers in extent and thus consists of an assemblage of many atoms. As depicted schematically in Figs. 1-3, a pin-jointed model that uses truss elements to represent the chemical bonds in the lattice structure can represent the RVE. To implement the resultant equivalent-truss structures, finite element models were developed by using ANSYS ${ }^{\circledR} 6$ (Ref. 31) (middle portion of Figs. 1-3). Each element (LINK8) was a three-dimensional pin-jointed truss element with six degrees of freedom (three displacement components on each end) that represented a single atomic interaction. Each node corresponded to an atom in the equilibrium structure of the molecular model. The Young's moduli of the truss elements were determined such that the total molecular potential energy of the molecular model and the strain energy of the equivalent-truss are equal for the same loading conditions. A total of 36,907 elements and 4751 nodes were used in the model of the nonfunctionalized nanotube, and a total of 36,481 elements and 4747 nodes was used for the model of the functionalized nanotube. For the crystalline polyethylene model, a total of 44,408 elements and 5469 nodes was used.

\section{Continuum Model}

With the equivalent-truss structure in place, the continuum models were constructed. The continuum model represents a key step in the homogenization process and is used to effectively define the structure-property relationships. The geometries of the homogeneous, equivalent-continuum RVEs were assumed to be cylindrical, similar to that of the molecular and truss models (right-hand side of Figs. 1-3). The mechanical properties of the continuum solid cylinders were determined by equating the total strain energies of the equivalent-truss and equivalent-continuum models under identical 
loading conditions. The rationale for determining the actual size of this cylindrical region is presented in detail in a subsequent section. The molecular model of the functionalized nanotube/polyethylene composite was assumed to exhibit orthotropic symmetry. There are nine independent material parameters required to determine the entire set of elastic constants for an orthotropic material, and each of the nine parameters can be determined from a single boundary condition applied to both equivalent-truss and equivalent-continuum models. For convenience, the nonfunctionalized and crystalline polyethylene models were also treated as orthotropic materials. Once the mechanical properties of the equivalent-continuum RVEs were determined, the two composite material RVEs were assumed to behave in the composite as effective fibers and were used in subsequent micromechanical analyses.

\section{Effective-Fiber Constitutive Model}

The constitutive relationship of an orthotropic equivalentcontinuum RVE (which is henceforth also referred to as an effective fiber) is

$$
\begin{gathered}
\sigma_{11}=C_{11}^{f} \varepsilon_{11}+C_{12}^{f} \varepsilon_{22}+C_{13}^{f} \varepsilon_{33} \\
\sigma_{22}=C_{12}^{f} \varepsilon_{11}+C_{22}^{f} \varepsilon_{22}+C_{23}^{f} \varepsilon_{33} \\
\sigma_{33}=C_{13}^{f} \varepsilon_{11}+C_{23}^{f} \varepsilon_{22}+C_{33}^{f} \varepsilon_{33} \\
\sigma_{23}=2 C_{44}^{f} \varepsilon_{23}, \quad \sigma_{13}=2 C_{55}^{f} \varepsilon_{13}, \quad \sigma_{12}=2 C_{66}^{f} \varepsilon_{12}
\end{gathered}
$$

where $\sigma_{i j}$ and $\varepsilon_{i j}$ are the stress and strain components, respectively $(i, j=1,2,3)$, and $C_{k l}^{f}$ are the elastic stiffness components of the effective fiber (denoted by superscript $f$ ), which are written in the usual contracted notation $(k, l=1, \ldots, 6)$.

Nine independent elastic properties are required to describe the overall elastic behavior of an orthotropic material, as shown in Eq. (1). For convenience, the nine independent elastic properties that can be used to describe the overall behavior of the effective fiber are the three elastic axial stiffness components $C_{11}^{f}, C_{22}^{f}$, and $C_{33}^{f}$; the three plane-strain bulk moduli $K_{23}^{f}, K_{13}^{f}$, and $K_{12}^{f}$; and the three elastic shear-stiffness components $C_{44}^{f}, C_{55}^{f}$, and $C_{66}^{f}$. The three plane-strain bulk moduli are defined as

$$
\begin{gathered}
K_{23}^{f}=\frac{1}{4}\left(C_{22}^{f}+C_{33}^{f}+2 C_{23}^{f}\right), \quad K_{13}^{f}=\frac{1}{4}\left(C_{11}^{f}+C_{33}^{f}+2 C_{13}^{f}\right) \\
K_{12}^{f}=\frac{1}{4}\left(C_{11}^{f}+C_{22}^{f}+2 C_{12}^{f}\right)
\end{gathered}
$$

where the subscripts indicate the plane that is subjected to a planestrain deformation. Equation (2) can be easily derived in a manner similar to the derivation of the transverse bulk modulus for a transversely isotropic material. ${ }^{24}$ Once the nine independent elastic properties are determined, the elastic interaction stiffness components $C_{23}, C_{13}$, and $C_{12}$ can be calculated from the relations in Eq. (2).

\section{Boundary Conditions}

At this point, the values of the nine elastic parameters are unknown. These values are determined by applying nine identical sets of boundary conditions to the equivalent-truss model and the effective fiber and by subsequently equating the strain energies by adjusting the nine independent elastic properties. Nine sets of boundary conditions were chosen to uniquely determine each of the nine independent elastic properties.

The displacements applied at the boundaries of the RVE are generalized by

$$
u_{i}(B)=\varepsilon_{i j} x_{j}
$$

where $B$ is the bounding surface, $x_{j}$ is defined in Figs. $1-3$, and the summation convention associated with repeated indices is used. The total strain energy of the effective fiber is

\begin{tabular}{|c|c|c|c|}
\hline Property & $\begin{array}{l}\text { Boundary } \\
\text { condition }\end{array}$ & $\begin{array}{c}\text { Boundary } \\
\text { displacement }\end{array}$ & $\begin{array}{l}\text { Strain } \\
\text { energy }\end{array}$ \\
\hline$C_{11}^{f}$ & $\varepsilon_{11}=e$ & $\begin{array}{l}u_{1}(B)=e x_{1} \\
u_{2}(B)=0 \\
u_{3}(B)=0\end{array}$ & $\Lambda^{f}=(V / 2) C_{11}^{f} e^{2}$ \\
\hline$C_{22}^{f}$ & $\varepsilon_{22}=e$ & $\begin{array}{l}u_{1}(B)=0 \\
u_{2}(B)=e x_{2} \\
u_{3}(B)=0\end{array}$ & $\Lambda^{f}=(V / 2) C_{22}^{f} e^{2}$ \\
\hline$C_{33}^{f}$ & $\varepsilon_{33}=e$ & $\begin{array}{l}u_{1}(B)=0 \\
u_{2}(B)=0 \\
u_{3}(B)=e x_{3}\end{array}$ & $\Lambda^{f}=(V / 2) C_{33}^{f} e^{2}$ \\
\hline
\end{tabular}

$$
\Lambda^{f}=(V / 2) \sigma_{i j} \varepsilon_{i j}
$$

The boundary conditions and strain energies for each of the nine independent elastic properties are listed in Tables 4-6. Unspecified strain components in Tables 4-6 are zero valued.
Table 4 Boundary conditions for axial stiffness components

Table 5 Boundary conditions for plane-strain bulk moduli

\begin{tabular}{lclc}
\hline \hline Property & $\begin{array}{c}\text { Boundary } \\
\text { condition }\end{array}$ & $\begin{array}{c}\text { Boundary } \\
\text { displacement }\end{array}$ & $\begin{array}{c}\text { Strain } \\
\text { energy }\end{array}$ \\
\hline$K_{23}^{f}$ & $\varepsilon_{22}=\varepsilon_{33}=e$ & $\begin{array}{l}u_{1}(B)=0 \\
u_{2}(B)=e x_{2}\end{array}$ & $\Lambda^{f}=2 V K_{23}^{f} e^{2}$ \\
& & $u_{3}(B)=e x_{3}$ & \\
$K_{13}^{f}$ & $\varepsilon_{11}=\varepsilon_{33}=e$ & $\begin{array}{l}u_{1}(B)=e x_{1} \\
u_{2}(B)=0\end{array}$ & $\Lambda^{f}=2 V K_{13}^{f} e^{2}$ \\
& & & \\
& & \\
$u_{3}(B)=e x_{3}$ & \\
$K_{12}^{f}$ & $\varepsilon_{11}=\varepsilon_{22}=e$ & $u_{1}(B)=e x_{1}$ \\
& & $u_{2}(B)=e x_{2}$ \\
& & \\
& & \\
& & \\
\hline
\end{tabular}

Table 6 Boundary conditions for shear-stiffness components

\begin{tabular}{lclc}
\hline \hline Property & $\begin{array}{c}\text { Boundary } \\
\text { condition }\end{array}$ & $\begin{array}{c}\text { Boundary } \\
\text { displacement }\end{array}$ & $\begin{array}{c}\text { Strain } \\
\text { energy }\end{array}$ \\
\hline$C_{44}^{f}$ & $\varepsilon_{23}=\gamma / 2$ & $\begin{array}{l}u_{1}(B)=0 \\
u_{2}(B)=(\gamma / 2) x_{3}\end{array}$ & $\Lambda^{f}=(V / 2) C_{44}^{f} \gamma^{2}$ \\
& & $u_{3}(B)=(\gamma / 2) x_{2}$ & \\
$C_{55}^{f}$ & $\varepsilon_{13}=\gamma / 2$ & $\begin{array}{l}u_{1}(B)=(\gamma / 2) x_{3} \\
u_{2}(B)=0\end{array}$ & $\Lambda^{f}=(V / 2) C_{55}^{f} \gamma^{2}$ \\
& & $u_{3}(B)=(\gamma / 2) x_{1}$ & \\
$C_{66}^{f}$ & $\varepsilon_{12}=\gamma / 2$ & $u_{1}(B)=(\gamma / 2) x_{2}$ & $\Lambda^{f}=(V / 2) C_{66}^{f} \gamma^{2}$ \\
& & $u_{2}(B)=(\gamma / 2) x_{1}$ & \\
& & \\
& & & \\
\hline
\end{tabular}

\section{Boundary Region}

The displacements specified in Tables 4-6 were applied to each node in the boundary region of the equivalent-truss model (indicated in Figs. 1-3), and the corresponding strain energies were calculated by summing the strain energies of each individual truss member in the RVE.

It was assumed that the thickness of the boundary region was equal to the maximum distance for which a positive-definite relationship exists between the force and displacement of all of the atom types. It was also assumed that the contribution of the energies associated with van der Waals forces between atoms with a larger distance than this maximum was relatively small and could be neglected.

Based on the repeating unit used in the MD simulation, the effective fiber radius and length were chosen to be 1.1 and $4.3 \mathrm{~nm}$, respectively. The calculated values of the nine independent parameters for the effective fibers with and without functionalization and the crystalline polyethylene material are listed in Table 7.

For the implementation of this modeling approach in this study, only static loading conditions were assumed. That is, the change in the equivalent-continuum material response as a function of time was not included, and it was therefore assumed that the material behaved in an elastic manner. Only the MD simulation included the effects of time, but only to establish a reliable RVE of the molecular models. 
Table 7 Equivalent-continuum properties (GPa)

\begin{tabular}{lccc}
\hline \hline Property & $\begin{array}{c}\text { Nonfunctionalized } \\
\text { effective fiber }\end{array}$ & $\begin{array}{c}\text { Functionalized } \\
\text { effective fiber }\end{array}$ & $\begin{array}{c}\text { Crystalline } \\
\text { polyethylene }\end{array}$ \\
\hline$C_{11}^{f}$ & 548.7 & 487.7 & 194.7 \\
$C_{22}^{f}$ & 16.8 & 24.5 & 3.6 \\
$C_{33}^{f}$ & 16.5 & 20.6 & 3.5 \\
$K_{23}^{f}$ & 14.8 & 19.5 & 2.6 \\
$K_{13}^{f}$ & 149.3 & 137.1 & 51.3 \\
$K_{12}^{f}$ & 149.2 & 138.7 & 52.0 \\
$C_{44}^{f}$ & 7.1 & 12.7 & 6.3 \\
$C_{55}^{f}$ & 144.0 & 155.4 & 5.1 \\
$C_{66}^{f}$ & 144.9 & 137.0 & 5.1 \\
\hline \hline
\end{tabular}

\section{Micromechanical Modeling}

Homogenization of a multiconstituent material requires the combination of the continuum method and a micromechanics model to provide a transition from the microscale to the macroscale. Micromechanics assumes small-deformation continuum mechanics as outlined in the preceding section. Continuum mechanics, in general, assumes uniform material properties within the boundaries of the problem. At the microscale, this assumption of uniformity might not hold, and hence the micromechanics method is used to express the continuum quantities associated with an infinitesimal material element in terms of the parameters that characterize the structure and properties of the microconstituents of the element. Constitutive models of the effective fiber/polymer composites were developed with a micromechanical analysis by using the mechanical properties of the nanotube/polymer effective fibers and the bulk polymer matrix material. For the composites considered in this study, the polymer molecules that were near the polymer/nanotube interface were included in the effective fiber, and it was assumed that the matrix polymer surrounding the effective fiber had mechanical properties equal to those of bulk polyethylene. Two types of polyethylene matricies were considered, an isotropic amorphous matrix and an anisotropic crystalline matrix. All relative movement and interaction of the polymer chains with respect to each other were modeled at the molecular level. This motion and interaction is therefore indirectly considered in the subsequent determination of the properties for the effective fibers, and it is therefore assumed that perfect bonding exists between the nanotube/polymer effective fibers and the surrounding polymer matrix in the micromechanics analysis.

A micromechanics method was used to predict the elastic mechanical properties of the composite material considered herein. ${ }^{32}$ For this method, the overall elastic-stiffness tensor of the composite containing orthotropic effective fibers embedded in a matrix material is

$$
\boldsymbol{C}=\boldsymbol{C}_{m}+c_{f}\left(\boldsymbol{C}_{f}-\boldsymbol{C}_{m}\right) \boldsymbol{A}_{f}
$$

where $\boldsymbol{A}_{f}$ is given by

$$
\boldsymbol{A}_{f}=\left[\boldsymbol{I}+\boldsymbol{S} \boldsymbol{C}_{0}^{-1}\left(\boldsymbol{C}_{f}-\boldsymbol{C}_{0}\right)\right]^{-1}
$$

where $S$ is the Eshelby tensor, ${ }^{33}$ which is given in detail elsewhere, ${ }^{34}$ and $\boldsymbol{C}_{0}$ is given by

$$
C_{k l}^{0}=C_{k l}^{m} \frac{1+\eta_{k l}^{f} c_{f}}{1-\eta_{k l}^{f} c_{f}}
$$

where

$$
\eta_{k l}^{f}=\frac{C_{k l}^{f}-C_{k l}^{m}}{C_{k l}^{f}+C_{k l}^{m}}
$$

In Eqs. (7) and (8), the usual contracted notation $(k, l=1, \ldots, 6)$ is employed, and there is no summation over repeated tensor subscripts. For unidirectional-aligned effective fibers, orientation averaging of Eq. (5) is not necessary, and the resulting elastic stiffness components of the composite have orthotropic symmetry. For threedimensional randomly oriented effective fibers, the orientation average of the stiffness tensor $\boldsymbol{C}$ is

$$
\langle\boldsymbol{C}\rangle=\left\langle C_{i j m n}\right\rangle=\left(\kappa-\frac{2}{3} \mu\right)\left(\delta_{i j} \delta_{m n}\right)+\mu\left(\delta_{i m} \delta_{j n}+\delta_{i n} \delta_{j m}\right)
$$

where $i, j, m, n=1,2,3$; the indicial summation convention is used; and

$$
\kappa=\frac{1}{9} C_{i i j j}, \quad \mu=\frac{1}{10}\left(C_{i j i j}-\frac{1}{3} C_{i i j j}\right)
$$

Therefore, from Eqs. (9) and (10), $\langle\boldsymbol{C}\rangle$ is isotropic.

Although it is convenient to establish constitutive equations of the composites in terms of the composite stiffness tensor $\boldsymbol{C}$, the Young's and shear moduli are the elastic constants that are most often used to compare the mechanical properties. Although the shear moduli of the composite material $\left(G_{44}, G_{55}, G_{66}\right)$ are simply equal to the shear-stiffness components $\left(C_{44}, C_{55}, C_{66}\right)$, the Young's moduli $\left(E_{1}\right.$, $\left.E_{2}, E_{3}\right)$ were calculated by using the components of the compliance tensor of the composite material, which was determined by inverting the composite stiffness tensor $\boldsymbol{C}$ (Ref. 35). The subscripts of the Young's moduli and shear moduli indicate the principal direction associated with the quantity, similar to Eq. (1). Therefore, $E_{1}$ is the longitudinal Young's modulus (parallel to the nanotube), $E_{2}$ and $E_{3}$ are transverse Young's moduli, $G_{44}$ is the transverse shear modulus, and $G_{55}$ and $G_{66}$ are longitudinal shear moduli. Because the random composites are isotropic, their mechanical properties are completely described by their Young's modulus $E\left(E=E_{1}=E_{2}=E_{3}\right)$ and shear modulus $G\left(G=G_{44}=G_{55}=G_{66}\right)$.

For the effective fiber/polymer composites considered in the present study, the elastic stiffness components, volume fraction, length, and orientation of the effective fiber were used for the fiber properties in Eq. (5). The effective fibers were assumed to have an spheroidal geometry for the Eshelby tensor, so that any length of effective fiber could be modeled. Although the nanotube and effective-fiber lengths are equivalent, the nanotube volume fraction was determined to be $52.9 \%$ of the effective-fiber volume fraction. This value was calculated by assuming the nanotube volume is defined as the total space occupied by the nanotube, including half of the unoccupied space between the nanotube and polymer. For the crystalline matrix, the properties of the crystalline polyethylene in Table 7 were used. The overall composite stiffness was calculated for effective-fiber lengths up to $450 \mathrm{~nm}$ and effective fiber volume fractions up to $90 \%$, which corresponds to the maximum volume fraction for hexagonally packed fibers. The calculations were performed assuming both perfectly aligned and three-dimensional randomly oriented effective fibers in both an amorphous and crystalline polyethylene matrix. The Eshelby tensor in Eq. (6) was evaluated numerically by using Gaussian quadrature. ${ }^{36}$

The amorphous polymer composite is modeled without directly applying the equivalent-continuum modeling technique to a nanotube/amorphous polymer system. Instead, the amorphous polymer composite is modeled with the effect fiber, which contains crystalline polyethylene molecules surrounding the nanotube, embedded in an amorphous polymer material. Therefore, for a more detailed study on the effects of nanotube functionalization on mechanical properties in completely amorphous, entangled polymer composite systems, the effective fiber should contain the nanotube and amorphous polymer chains.

\section{Results and Discussion}

In this section, the elastic constants of the three equivalentcontinuum models are compared and discussed. The moduli of the nanotube composites, as predicted using the micromechanics technique just described, are also presented in terms of nanotube length, volume fraction, and orientation.

\section{Equivalent-Continuum Models}

In Table 7, it is clear that functionalization had a measurable effect on the elastic properties of the effective fibers. Although functionalization reduced the longitudinal elastic constant $C_{11}^{f}$ by 
$11 \%$, it enhanced the transverse elastic constants $C_{22}^{f}$ and $C_{33}^{f}$ by 46 and $25 \%$, respectively. Similarly, the longitudinal plane-strain bulk moduli $K_{13}^{f}$ and $K_{12}^{f}$ were reduced by functionalization by 8 and $7 \%$, respectively, whereas the transverse plane-strain bulk modulus $K_{23}^{f}$ was increased by $3 \%$. Functionalization changed the longitudinal shear moduli $C_{55}^{f}$ and $C_{66}^{f}$ by +8 and $-6 \%$, respectively. The transverse shear modulus $C_{44}^{96}$ was enhanced by $79 \%$ with the functionalization. At the length scale of the effective fiber, it appears that the key benefit of functionalization in nanotube/polymer composites is a significant gain in transverse elastic properties. This gain comes at the expense of smaller losses in the longitudinal properties.

The properties of the crystalline polyethylene material are provided in Table 7. Clearly the elastic properties of the polyethylene are lower than those of the nanotube/polyethylene effective fibers, indicating a definite reinforcing effect of nanotubes. The properties of the crystalline polymer (Table 7) are bounded by those found in the literature obtained by experimental and analytical techniques. ${ }^{37,38}$ The literature values of the mechanical properties show considerable variation because of the difficulties of obtaining the properties of crystalline polymers via experimental and analytical techniques. ${ }^{39,40}$

\section{Elastic Properties of Composites}

The calculated Young's moduli of the nanotube composites with the amorphous matrix are plotted in Fig. 5 as a function of nanotube length, for a $1 \%$ nanotube volume fraction. The longitudinal Young's modulus of the aligned composite and the Young's modulus of the random composite had a nonlinear dependence on the nanotube length. An increase in the Young's modulus with respect to an increase in nanotube length is expected to correspond to a relative increase in load transfer efficiency between the nanotube and the surrounding polymer. The data in Fig. 5 indicate that at a nanotube length of about $400 \mathrm{~nm}$, the efficiency of load transfer is nearly maximized, as evidenced by the nearly zero slope in the data curve. Further increases in nanotube length beyond $400 \mathrm{~nm}$ result in relatively small increases in Young's modulus for a given nanotube volume fraction. As the nanotube length became greater than approximately $100 \mathrm{~nm}$, the Young's modulus for the composite without nanotube functionalization became larger than that of the composite with nanotube functionalization for the random composite and longitudinal deformation of the aligned composite. At $450 \mathrm{~nm}$, the functionalization reduced the longitudinal Young's modulus of the aligned composite and the Young's modulus of the random composite by 11 and $7 \%$, respectively. In contrast, the transverse Young's modulus of the aligned composite had no dependence on nanotube length. Also, there was no effect of functionalization on the transverse deformation of the aligned composite.

The shear moduli of the nanotube composites with the amorphous matrix are plotted in Fig. 6 as a function of nanotube length for a $1 \%$ nanotube volume fraction. The shear modulus of the random composite had a nonlinear dependence on nanotube length, whereas the

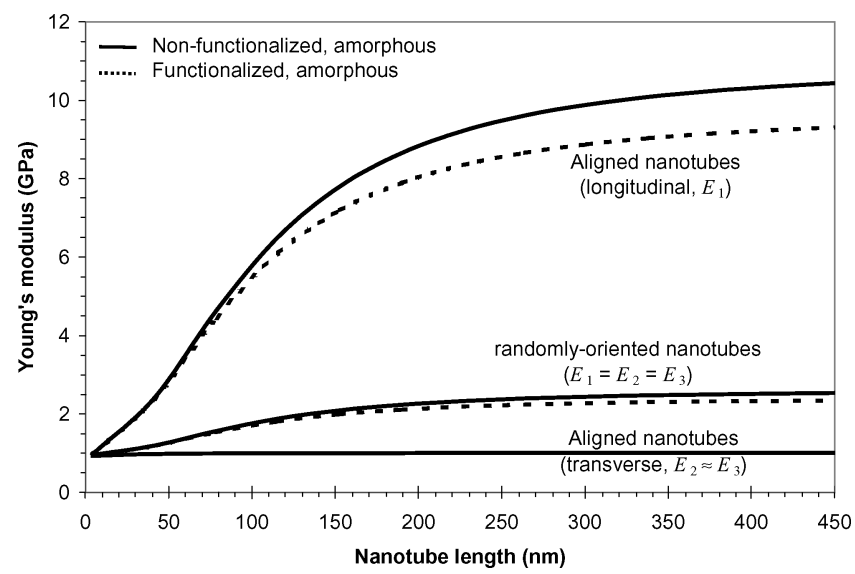

Fig. 5 Young's moduli $E_{i}$ of the composite systems vs nanotube length for a $1 \%$ nanotube volume fraction.

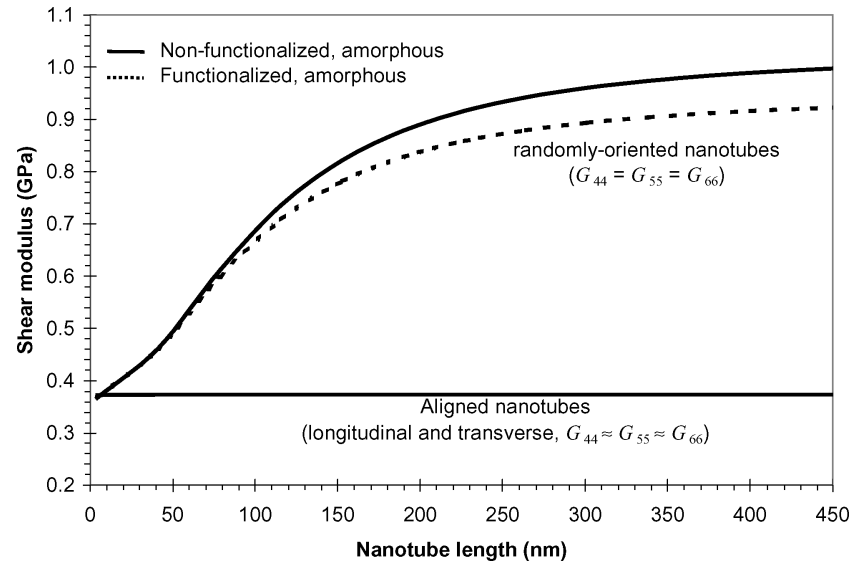

Fig. 6 Shear moduli $G_{i j}$ of the composite systems vs nanotube length for a $1 \%$ nanotube volume fraction.

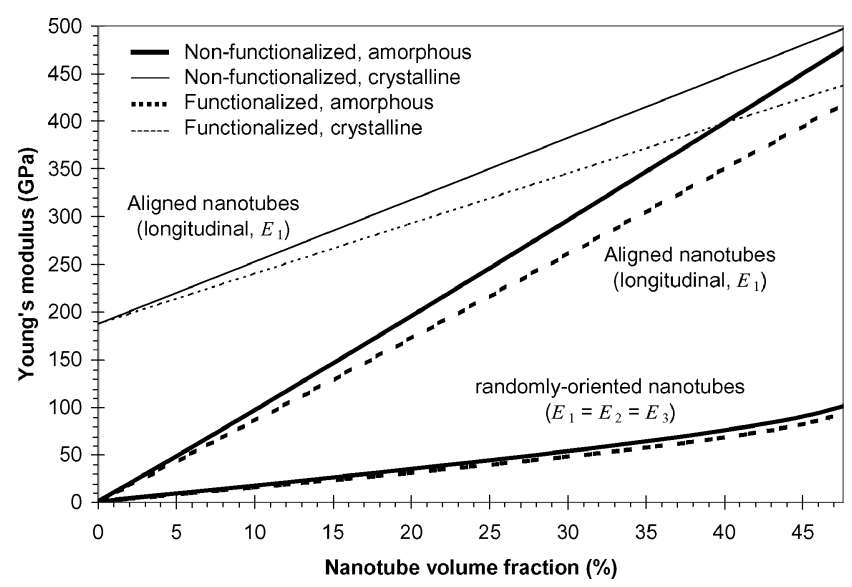

Fig. 7 Longitudinal Young's moduli $E_{1}$ of the composite systems vs nanotube volume fraction for a nanotube length of $400 \mathrm{~nm}$.

shear modulus of the aligned composite had no dependence on nanotube length. Again, the data indicate that the efficiency of load transfer, as evidenced by a modulus change, is nearly maximized at a nanotube length of about $400 \mathrm{~nm}$. As the nanotube length became greater than approximately $100 \mathrm{~nm}$, the shear modulus for the nonfunctionalized nanotube composite became larger than that of the functionalized nanotube composite for the random composite. At $450 \mathrm{~nm}$, the functionalization reduced the Young's modulus of the random composite by $8 \%$. There was no significant effect of functionalization on the shear modulus of the aligned composite as the nanotube length was increased.

The longitudinal Young's moduli of the random and aligned composites with both amorphous and crystalline matrices are plotted in Fig. 7 as a function of nanotube volume fraction, for a constant nanotube length of $400 \mathrm{~nm}$. At this nanotube length, the change in the longitudinal Young's modulus with respect to nanotube volume fraction of the aligned composite was nearly linear, resembling a linear rule-of-mixtures relationship. Over the complete range of nanotube volume fraction, the functionalization of the nanotube reduced the longitudinal Young's modulus of all three composites.

The transverse Young's moduli of the aligned composites with both amorphous and crystalline matrices are plotted in Fig. 8 as a function of nanotube volume fraction, for a constant nanotube length of $400 \mathrm{~nm}$. In contrast to the Young's moduli in Fig. 7, the transverse Young's moduli of both composites improved when the nanotubes were functionalized. This result is consistent with the improvement in the transverse properties of the functionalized nanotube effective fiber. The enhancement is evident for nanotube volume fractions greater than $10 \%$ in both composites.

The longitudinal shear moduli of the composites with both amorphous and crystalline matrices are plotted in Fig. 9 as a function 


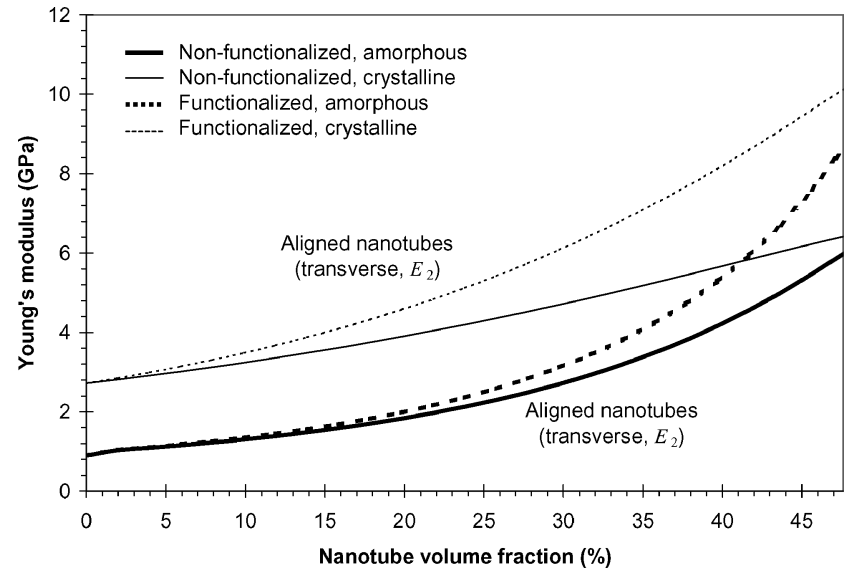

Fig. 8 Transverse Young's moduli $E_{2}$ of the composite systems vs nanotube volume fraction for a nanotube length of $400 \mathrm{~nm}$.

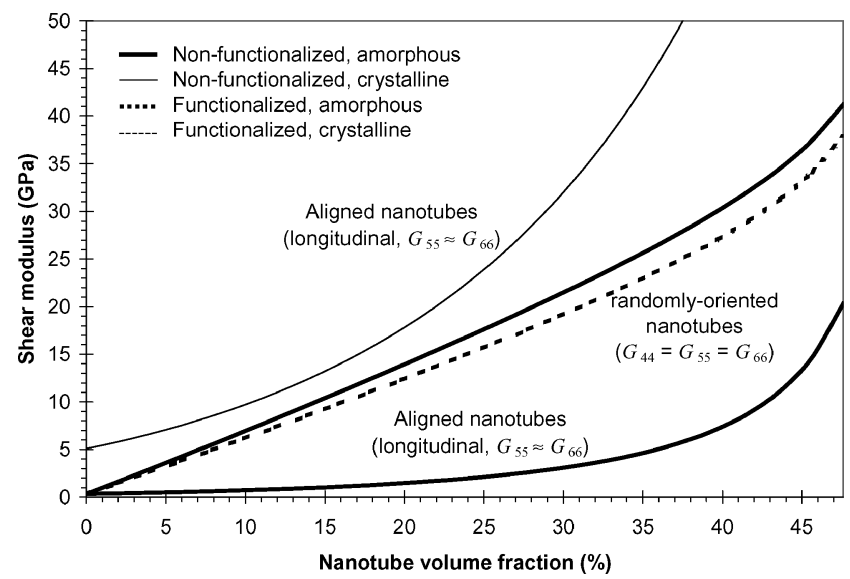

Fig. 9 Longitudinal shear moduli $G_{55}$ and $G_{66}$ of the composite systems vs nanotube volume fraction for a nanotube length of $400 \mathrm{~nm}$.

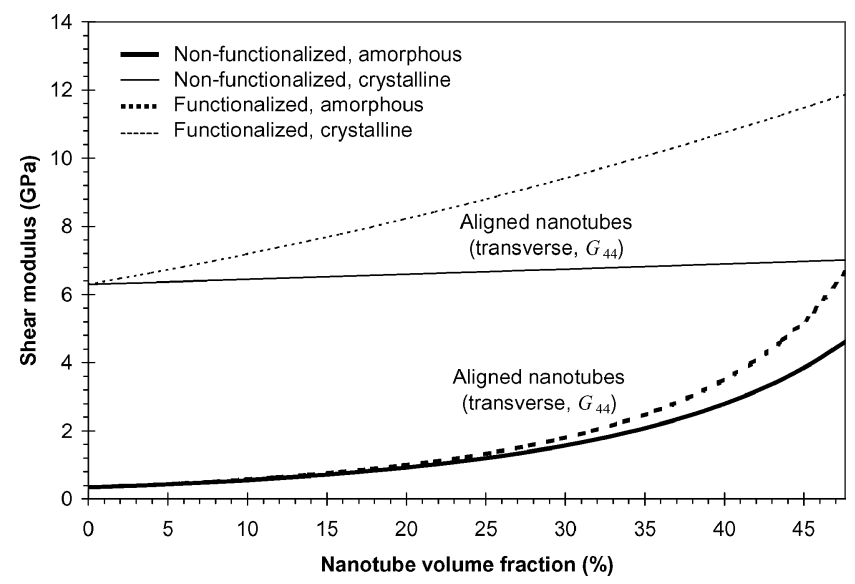

Fig. 10 Transverse shear moduli $G_{44}$ of the composite systems vs nanotube volume fraction for a nanotube length of $400 \mathrm{~nm}$.

of nanotube volume fraction. As the nanotube volume fraction increased, the functionalization reduced the shear modulus of the randomly oriented nanotube composite with the amorphous matrix. However, there was no significant effect of the functionalization on the shear moduli of the aligned composites with amorphous and crystalline matrices for the given range of nanotube volume fractions.

The transverse shear moduli of the composites with both amorphous and crystalline matrices are plotted in Fig. 10 as a function of nanotube volume fraction. As the nanotube volume fraction increased, the functionalization improved the transverse shear modulus of both composites. The improvement was particularly noticeable for the composite with the crystalline matrix at large nanotube volume fractions. This trend is very similar to volume fraction dependence of the transverse Young's moduli of the same composites.

\section{Conclusions}

In this study, the bulk elastic properties of a single-walled carbon nanotube/polyethylene composite have been predicted for both functionalized and nonfunctionalized nanotubes in amorphous and crystalline polyethylene matrices using a hierarchical approach based on the equivalent continuum method. It was assumed that functionalization would affect the load transfer between the nanotube and the surrounding matrix and that changes in predicted elastic stiffness could be directly related to the efficiency of the load-transfer mechanism. The effective continuum analysis method established constitutive equations for both functionalized and nonfunctionalized nanotube composites systems by using a hierarchical modeling technique that predicted elastic bulk behavior, using intrinsic properties developed through molecular dynamics simulations. The elastic properties of the composites with and without nanotube functionalization have been examined for various nanotube lengths, volume fractions, nanotube orientations, and both amorphous and crystalline polyethylene matrices.

For a fixed nanotube volume fraction of $1 \%$ and various nanotube lengths, the Young's and shear moduli of the random composites and the longitudinal Young's moduli of the aligned composites have been shown to decrease up to $11 \%$ when the nanotube is functionalized. For a fixed nanotube length of $400 \mathrm{~nm}$ and various nanotube volume fractions, the longitudinal Young's moduli of the aligned composites and both the Young's and shear moduli of the random composites have also been shown to decrease up to $12 \%$ when the nanotube is functionalized. However, under these conditions the transverse Young's moduli and the transverse shear moduli of the aligned composites have shown an increase of up to $45 \%$ when the nanotube is functionalized.

The primary implication of these results is that although chemical functionalization of single-walled carbon nanotubes has been considered as a means to increase load-transfer efficiency in a nanotube/polymer composite, this functionalization has, in fact, degraded most of the macroscopic elastic stiffness components of the composite materials considered in this study. In general, this occurs for both random composites and aligned composites with both amorphous and crystalline matrices over a wide range of nanotube volume fractions and lengths.

\section{References}

${ }^{1}$ Thostenson, E. T., Ren, Z., and Chou, T. W., "Advances in the Science and Technology of Carbon Nanotubes and Their Composites: A Review," Composites Science and Technology, Vol. 61, No. 13, 2001, pp. 1899-1912.

${ }^{2}$ Sun, Y. P., Fu, K., Lin, Y., and Huang, W., "Functionalized Carbon Nanotubes: Properties and Applications," Accounts of Chemical Research, Vol. 35, No. 12, 2002, pp. 1096-1104.

${ }^{3}$ Chen, J., Hamon, M. A., Hu, H., Chen, Y., Rao, A. M., Eklund, P. C., and Haddon, R. C., "Solutions Properties of Single-Walled Carbon Nanotubes," Science, Vol. 282, No. 5386, 1998, pp. 95-98.

${ }^{4}$ Bahr, J. L., and Tour, J. M., "Highly Functionalized Carbon Nanotubes Using In Situ Generated Diazonium Compounds," Chemistry of Materials, Vol. 13, No. 11, 2001, pp. 3823, 3824.

${ }^{5}$ Mickelson, E. T., Huffman, C. B., Rinzler, A. G., Smalley, R. E., Hauge, R. H., and Margrave, J. L., "Fluorination of Buckytubes," Chemical Physics Letters, Vol. 296, No. 1-2, 1998, pp. 188-194.

${ }^{6}$ Mickelson, E. T., Chiang, I. W., Zimmerman, J. L., Boul, P. J., Lozano, J., Liu, J., Smalley, R. E., Hauge, R. H., and Margrave, J. L., "Solvation of Fluorinated Single-Wall Carbon Nanotubes in Alcohol Solvents," Journal of Physical Chemistry B, Vol. 103, No. 21, 1999, pp. 4318-4322.

${ }^{7}$ Boul, P. J., Liu, J., Michelson, E. T., Huffman, C. B., Ericson, L. M., Chiang, I. W., Smith, K. A., Colbert, D. T., Hauge, R. H., Margrave, J. L., and Smalley, R. E., "Reversible Sidewall Functionalization of Buckytubes," Chemical Physics Letters, Vol. 310, No. 3-4, 1999, pp. 367-372.

${ }^{8}$ Chen, Y., Haddon, R. C., Fang, S., Rao, A. M., Eklund, P. C., Lee, W. H., Dickey, E. C., Grulke, E. A., Pendergrass, J. C., Chavan, A., Haley, B. E., and Smalley, R. E., "Chemical Attachment of Organic Functional Groups to 
Single-Walled Carbon Nanotube Material," Journal of Materials Research, Vol. 13, No. 9, 1998, pp. 2423-2431.

${ }^{9}$ Jin, Z., Sun, X., Xu, G., Goh, S. H., and Ji, W., "Non-Linear Optical Properties of Some Polymer/Multi-Walled Carbon Nanotube Composites," Chemical Physics Letters, Vol. 318, No. 6, 2000, pp. 505-510.

${ }^{10}$ Hill, D. E., Lin, Y., Rao, A. M., Allard, L. F., and Sun, Y. P., "Functionalization of Carbon Nanotubes with Polystyrene," Macromolecules, Vol. 35, No. 25, 2002, pp. 9466-9471.

${ }^{11}$ Lin, Y., Rao, A. M., Sadanadan, B., Kenik, E. A., and Sun, Y. P., "Functionalizing Multiple-Walled Carbon Nanotubes with Aminopolymers," Journal of Physical Chemistry B, Vol. 106, No. 6, 2002, pp. 1294-1298.

${ }^{12}$ Huang, W., Taylor, S., Fu, K., Lin, Y., Zhang, D., Hanks, T. W., Rao, A. M., and Sun, Y. P., "Attaching Proteins to Carbon Nanotubes via DiimideActivated Amidation," NanoLetters, Vol. 2, No. 4, 2002, pp. 311-314.

${ }^{13}$ Velasco-Santos, C., Martinez-Hernandez, A. L., Lozada-Cassou, M., Alvarex-Castillo, A., and Castano, V. M., "Chemical Functionalization of Carbon Nanotubes Through an Organosilane," Nanotechnology, Vol. 13, No. 4, 2002, pp. 495-498.

${ }^{14}$ Banerjee, S., and Wong, S. S., "Structural Characterization, Optical Properties, and Improved Solubility of Carbon Nanotubes Functionalized with Wilkinson's Catalyst," Journal of the American Chemical Society, Vol. 124, No. 30, 2002, pp. 8940-8948.

${ }^{15}$ Chen, R. J., Zhan, Y. G., Wang, D., and Dai, H., "Noncovalent Sidewall Functionalization of Single-Walled Carbon Nanotubes for Protein Immobilization," Journal of the American Chemical Society, Vol. 123, No. 16, 2001, pp. 3838-3839.

${ }^{16}$ Star, A., Stoddart, J. F., Steuerman, D., Diehl, M., Boukai, A., Wong, E. W., Yang, X., Chung, S., Choi, H., and Heath, J. R., "Preparation and Properties of Polymer-Wrapped Single-Walled Carbon Nanotubes," Angewandte Chemie International Edition in English, Vol. 40, No. 9, 2001, pp. $1721-1725$

${ }^{17}$ Lordi, V., and Yao, N., "Molecular Mechanics of Binding in CarbonNanotube-Polymer Composites," Journal of Materials Research, Vol. 15, No. 12, 2000, pp. 2770-2779.

${ }^{18}$ Jia, Z., Wang, Z., Xu, C., Liang, J., Wei, B., Wu, D., and Zhu, S., "Study on Poly(Methyl Methacrylate)/Carbon Nanotube Composites," Materials Science and Engineering A, Vol. A271, No. 1-2, 1999, pp. 395-400.

${ }^{19}$ Wagner, H. D., Lourie, O., Feldman, Y., and Tenne, R., "Stress-Induced Fragmentation of Multiwall Carbon Nanotubes in a Polymer Matrix," Applied Physics Letters, Vol. 72, No. 2, 1998, pp. 188-190.

${ }^{20}$ Georgakilas, V., Kordatos, K., Prato, M., Guldi, D. M., Holzinger, M., and Hirsch, A., "Organic Functionalization of Carbon Nanotubes," Journal of the American Chemical Society, Vol. 124, No. 5, 2002, pp. 760, 761.

${ }^{21}$ Georgakilas, V., Voulgaris, D., Vazquez, E., Prato, M., Guldi, D. M., Kukovecz, A., and Kuzmany, H., "Purification of HiPCO Carbon Nanotubes via Organic Functionalization," Journal of the American Chemical Society, Vol. 124, No. 48, 2002, pp. 14,318, 14,319.

${ }^{22}$ Frankland, S. J. V., Caglar, A., Brenner, D. W., and Griebel, M., "Molecular Simulation of the Influence of Chemical Cross-Links on the Shear Strength of Carbon Nanotube-Polymer Interfaces," Journal of Physical Chemistry B, Vol. 106, No. 28, 2002, pp. 3046-3048.

${ }^{23}$ Odegard, G. M., Gates, T. S., Nicholson, L. M., and Wise, K. E., "Equivalent-Continuum Modeling of Nano-Structured Materials," Composites Science and Technology, Vol. 62, No. 14, 2002, pp. 1869-1880.
${ }^{24}$ Odegard, G. M., Gates, T. S., Wise, K. E., Park, C., and Siochi, E. J., "Constitutive Modeling of Nanotube-Reinforced Polymer Composites," Composites Science and Technology, Vol. 63, No. 11, 2003, pp. 1671-1687.

${ }^{25}$ Sperling, L. H., Introduction to Physical Polymer Science, Wiley, New York, 1992.

${ }^{26}$ Rappe, A. K., and Casewit, C. J., Molecular Mechanics Across Chemistry, Univ. Science Books, Sausalito, CA, 1997.

${ }^{27}$ Brenner, D. W., Shenderova, O. A., Harrison, J. A., Stuart, S. J., Ni, B., and Sinnott, S. B., "Second Generation Reactive Empirical Bond Order (REBO) Potential Energy Expression for Hydrocarbons," Journal of Physics C: Condensed Matter, Vol. 14, No. 4, 2002, p. 783.

${ }^{28}$ Allen, M. P., and Tildesley, D. J., Computer Simulation of Liquids, Oxford Univ. Press, Oxford, 1987.

${ }^{29}$ Smith, W., and Forester, T. R., DL-POLY, Council for the Central Lab. of the Research Councils, Warrington, England, U.K., 1996.

${ }^{30}$ Cornell, W. D., Cieplak, P., Bayly, C. I., Gould, I. R., Merz, K. M., Ferguson, D. M., Spellmeyer, D. C., Fox, T., Caldwell, J. W., and Kollman, P. A., "A Second Generation Force Field for the Simulation of Proteins, Nucleic Acids, and Organic Molecules," Journal of the American Chemical Society, Vol. 117, No. 19, 1995, pp. 5179-5197.

${ }^{31}$ ANSYS, Ver. 6, SAS IP, Canonsburg, PA, Aug. 2001.

${ }^{32}$ Odegard, G. M., "Constitutive Modeling of Piezoelectric Polymer Composites," Acta Materialia, Vol. 52, No. 18, 2004, pp. 5315-5330.

${ }^{33}$ Eshelby, J. D., "The Determination of the Elastic Field of an Ellipsoidal Inclusion, and Related Problems," Proceedings of the Royal Society of London, Series A, Vol. 241, 1957, pp. 376-396.

${ }^{34}$ Mura, T., Micromechanics of Defects in Solids, Martinus Nijhoff, The Hague, The Netherlands, 1982.

${ }^{35}$ Hull, D., and Clyne, T. W., An Introduction to Composite Materials, Cambridge Univ. Press, Cambridge, England, U.K., 1996, Chap. 5.

${ }^{36}$ Davis, P. J., and Polonsky, I., "Numerical Interpolation, Differentiation and Integration," Handbook of Mathematical Functions with Formulas, Graphs, and Mathematical Tables, edited by M. Abramowitz and I. A. Stegun, Wiley, New York, 1972, pp. 875-924.

${ }^{37}$ Holliday, L., and White, J. W., "The Stiffness of Polymers in Relation to Their Structure," Pure and Applied Chemistry, Vol. 26, No. 3-4, 1971, pp. 545-582.

${ }^{38}$ Lacks, D. J., and Rutledge, G. C., "Simulation of the Temperature Dependence of Mechanical Properties of Polyethylene," Journal of Physical Chemistry, Vol. 98, No. 4, 1994, pp. 1222-1231.

${ }^{39}$ Al-Hussein, M., Davies, G. R., and Ward, I. M., "Mechanical Properties of Oriented Low-Density Polyethylene with an Oriented Lamellar-Stack Morphology," Journal of Polymer Science: Part B: Polymer Physics, Vol. 38, No. 5, 2000, pp. 755-764.

${ }^{40}$ Van Dommelen, J. A. W., Parks, D. M., Boyce, M. C., Brekelmans, W. A. M., and Baaijens, F. P. T., "Micromechanical Modeling of Intraspherulitic Deformation of Semicrystalline Polymers," Polymer, Vol. 44, No. 19, 2003, pp. 6089-6101.
K. Shivakumar Associate Editor 